\title{
Adaptive Cost Accounting Control: Issues in Realizing Deming Synergy
}

\author{
Mohamed Gaber ${ }^{1}$, Edward J. Lusk ${ }^{1}$ \\ ${ }^{1}$ The State University of New York (SUNY) at Plattsburgh, Plattsburgh, NY, USA \\ Correspondence: Edward J. Lusk, The State University of New York (SUNY) at Plattsburgh, Plattsburgh, NY, \\ USA.
}

Received: December 8, 2017

Accepted: January 26, $2018 \quad$ Online Published: February 12, 2018

doi:10.5539/ibr.v11n3p76

URL: https://doi.org/10.5539/ibr.v11n3p76

\begin{abstract}
We report on a consultation addressing the re-configuration of a Standards Cost Accounting System of a major MNC. We identified two fundamental theoretical issues pertinent to this re-configuration: Their Standards Cost Accounting [SCA] System was (1) not adaptive within their control time frame, and (2) the holistic systemic protocols espoused by W. Edwards Deming were not used to condition the decision-making framework addressing control. We developed an adaptive Decision Support System [SCA:DSS] that offered the following integrated systemic features: (i) The SCA:DSS is parametrized using the Marketing/Sales sub-budget as approved by corporate-level management and (ii) is used to set the control standards for direct Materials \& Labor costs and $\mathrm{ABC}$ related allocations, (iii) A detailed interactive profiling of production activity is produced at a time when adaptive corrective actions would still be reasonably possible, and (iv) Adaptive: Best, Stasis and Corrective Action Cases regarding the effect of these corrective actions on the contribution margin are displayed. However, even given the adaptive design features and the explicit designs to effect holistic integration over the pilot division and the central headquarters of the firm, the SCA: DSS failed to be implemented. We offer valuable insights into this failure-to-launch that may be indispensable in effecting a synergetic environment where adaptive holistic cost control may be realized. In this paper all of the technical functionalities of the SCA: DSS are detailed and a working illustration is provided. The SCA: DSS is offered as a free download without restrictions to its use.
\end{abstract}

Keywords: variance analysis, Deming, holistic

\section{Introduction: The Theoretical Design Context for Adaptive Control}

It is interesting that most of the decision-making systemic re-parametrizations regarding cost control are done on a yearly basis. Simply, learning and adaptive control features are usually formed over the accounting cycle that follows the audit year. The reason for this is likely due to the fact there are no Cost Accounting textbooks, of which we are aware, that address in practical detail the interactive intra-annual systemic holistic aspects of cost control. For example, consider the latest Horngren text treatment on Cost Control (Datar \& Rajan, 2018) - clearly one of the trend-setting text presentations over the last 40 years. This widely adopted text from Pearson[https://www.pearson.com/us/], one of the market leaders in the academic text marketplace, offers the following council:

Planning consists of selecting an organization's goals and strategies, predicting results under various alternative ways of achieving those goals, deciding how to attain the desired goals, and communicating the goals and how to achieve them to entire organization. (p.10)

Control comprises taking that implement the planning decisions, evaluating past performance, and providing feedback and learning to help future decision making. (p.10)

They continue;

Planning and Control activities must be flexible enough so that managers can seize opportunities unforeseen at the time the plan was formulated. In no case should control mean that managers cling to a plan when unfolding events (---) indicate that actions not encompassed by that plan (---) would offer better results for the company. (p.11)

Benchmarking is the continuous process of comparing your firm's performance levels against the 
best levels of performance in competing companies or in companies having similar processes. When benchmarks are used as standards, managers and management accountants know that the company will be competitive in the marketplace if it can met or beat the standards. (pp.267-8)

As "spot-on" as this advice is relative to encouraging decision-makers [DM] to be attentive to effecting adaptive and holistic cost control, there are no detailed practical illustrative examples of adaptive control and further the Deming $(1992,1993)$ protocols, as such, are not referenced or illustrated in the text.

This is the point of departure of our research presentation. We are offering an interesting and, as far as we can determine from the literature in Cost Accounting focused on process control, a unique modeling perspective that is programmed in an Excel ${ }^{\mathrm{TM}}$ open-access VBA/Modular Decision Support System called the Standards Cost Accounting DSS [SCA:DSS ${ }^{\mathrm{i}}$ ]. Following we will:

1. Discuss the theoretical context for the consulting engagement that led to the development of the SCA:DSS for production cost monitoring for control.

2. Present in detail the three principal elements of the SCA: DSS: (i) Adaptive-Integrated Cost Control respecting Net Planned Controllable Cost Contribution Margin, (ii) Determination of the reduction of the Controllable Process Costs needed to achieve the a-priori Contribution Margin Goals, and (iii) A Best Case, A Status-Quo or Stasis Case, and an Iterative Case profile that underlie the What-If decision platform.

3. Offer a comprehensive example that was used in the presentation to the client of the functioning of the SCA: DSS.

4. Present debrief reflections on the consulting engagement and offer extensions of the SCA: DSS that will enhance its practical utility.

\section{The Development of a System for Cost Evaluation for Purposes of Control: The Theoretical Imperatives}

\subsection{Literature Context for Adaptive \& Holistic Control}

Indeed it is interesting from a practical perspective that such obvious paradigms as: Adaptive Real-Time Cost Control and Holistic integration of the decision-making logistics, which have their roots in Ackoff's (1967) early work introduced in Management Misinformation Systems and later re-formed and exquisitely detailed by Deming $(1992,1993)$ are not presented in practical detail and extensively illustrated in excellent texts such as the Horngren work or the like. To elaborate; these two paradigms are usually not in focal view of those informing managers responsible for the design and execution of cost control platforms. Let us consider these two essential theoretic guidelines as they will form the nexus of our work.

\subsubsection{Adaptive Control}

There are three recent works that provide excellent contextual evidence of the necessity of maintaining an adaptive functionality for dynamic control systems. Linnenluecke, Birt \& Griffiths (2015) demonstrate in a research context that being adaptive is the design imperative in addressing: (i) risk calibration by assessing vulnerability and the capacity for corrective reaction, and (ii) integrated overall organizational valuation by balancing cost and related benefits. In a related context which highlights the importance of systemic coordination needed to capture the possibility of synergy in a complex logistical context, Zugno \& Conejo (2015) note that their system's model that was organized to address various control aspects dealing with component and overall uncertainly could only achieve its unified goals though adaptive controls that were dynamic in the real time execution of the modeling protocols. Finally, as a cogent summary underscoring the necessity of adaptive systemic controls in a dynamic context Brimson, J. (2011, p.33) notes:

In an adaptive management system, the nexus of decision making is shifted from fixed-period planning and management to a management of revenue, structural, and discretionary cost based on the current events at the analysis date.

\subsubsection{The Deming Holistic Imperative}

Following we offer a capsule of the holistic imperatives that is due to both Ackoff (1967) and Deming (1992, 1993). This will be relevant in understanding the systemic issues raised in executing adaptive controls. Denzau, Minassians \& Roy. (2016) offer here paraphrased:

W. Edwards Deming asserted that if actors operating in discrete agencies are to communicate and cooperate across organizational boundaries to get things done, they must shift their focus from an individual departmental or organizational level to a systems level_known as systems thinking. 
According to Deming, a system is: A series of functions of activities, sub-processes or stages that exist both within and external to a given organization that must all work together to accomplish a common aim. (Deming (2005), p.90). Successful management of an organization, therefore, requires that agency leaders possess a working 'knowledge of the interrelationships between all of the components and sub-processes within the system and everybody that works in it'.

We will be reporting on a consultation as this will provide many insights of value regarding the difficulties of effecting adaptive control in the systemic context. The nature of our report follows the work of Venzor \& Ivanov (2017) who also report on the practical context of their interactions in a corporate context [A successful Fast-food franchise with nine franchise agents]. Pertaining to the importance of acceptance of the need to find a "satisficing" action plan in the dynamic of the organizational milieu regarding adaptive control, they note (p. 108):

According to Deming, managing by results is "when you take immediate action on any fault, defect, complaint, delay, accident, or breakdown" (Deming, 1992, 1993). When using management by results, you find an immediate pseudo-solution opposed to identifying the root of the problem.

We will see this advice play out in the context of our report where in the adaptive context there may be multiple options and the DM must select one that is in the proximal temporal content rather than engage an examination of the best action plan considering all the aspects of the problem. Simply, there is rarely time to effect such a strategy search. However, this should not be confused with Deming's advice to consider the temporal context. Deming notes that short-term solutions have long-term effects. This advice in adaptive systems requires that DM consider the counteracting and countervailing aspects of the corrective actions. Specifically, in a dynamic adaptive context, effectively, DM try to identify those actions that could conform to the adaptive nature of the problem and within this set of possible actions which one provides acceptable flexibility for future actions.

In this regard, following the holistic imperatives of Deming the usual characteristics of production process accounting control that we found to be relevant in this context for the adaptive nature of the holistic system are: (i) systems-wide decision-making integration over the various activity centers and agents that impact and are impacted by the firm's production process, (ii) reliable and relevant benchmarks for variance identification for selected cost components, (iii) timely, accurate and consistent measures of production activity, (iv) timely feedback to those responsible and empowered to take corrective actions, (vi) protocol guidance for taking corrective actions that are coordinated with the reward protocol(s) for the agents in the system, and finally (vii) the post-corrective action-reevaluation of the control actions are part of the learning feedback loop. This monitoring for evaluation and control cycle then is re-engaged in the next planning cycle. This is the theoretical underpinning of control theory as applied to the accounting process control and has been used to guide the development of the SCA:DSS. However, the commitment to process control is also critical in the context of the certified audit. In this regard, the external auditors are indeed interested in the client's efficient and effective utilization of resources as this is, in some cases, a Going Concern issue that falls under the purview of the Public Company Accounting Oversight Board and so also of the Security \& Exchange Commission. Finally, as suggested by Babicky (1996), Certified Public Accountants in their advisory role to the management of firms that are using process production configurations are also keenly interested in the use of DSS to facilitate the corrective actions that are often needed to maintain control over the production process. This is indeed a large domain of possible interest and will form our investigative context.

\section{The Study Context and the Principal Functionalities of the SCA: DSS}

Recall our study reports on a consulting engagement conducted for a division of a Multinational Corporation $[\mathrm{MNC}]$ that produced precision-measuring devices many of which were products sold to firms to enable them to control their production processes. The deliverable for this academic consultation-meaning that there was no fee charged to the division and some of the work was done by students as supervised by a faculty member - was a Standard Cost Accounting Decision Support System [SCA:DSS] programmed in Excel. The faculty member collected all the data \& information used in this study, had oversight of the development of the SCA:DSS, and had access to the divisional Accounting Information System [AIS] data and support from the divisional Chief Financial Officer.

\subsection{Product Profile}

The division produced essentially one major physical product which was a programming shell [PS]—a production control router configuration of sorts. The PS was sold essentially with the intention that most of the control software would be purchased from the MNC and uploaded/installed to the programming shell. The customers could/would then make the programming modifications that suited their needs. This necessitated that 
the PS had and maintained compatibility links with the current versions of: Excel ${ }^{\mathrm{TM}}$, SAP ${ }^{\mathrm{TM}}$, SAS ${ }^{\mathrm{TM}}$, C ${ }^{\mathrm{TM}} \&$ $\mathrm{C}^{+} \mathrm{TM}$ and Java ${ }^{\mathrm{TM}}$.

\subsection{Market \& Cost Integration}

As emphasized by Parnaby \&Towill (2009) the divisional cost planning process should be aligned with the market return of the product base. This is critical in the Deming content as it links the various component aspects of the organization into the decision-making milieu. In this way, there is a natural check on contribution margin control and so possibly with the coordination of the overall profitability of the various decision agents in the system. This was to some extent the case for this MNC. Specifically, the Sales Division proposes: The gross market price of the products for the first two quarters of the year. There are a variety of modifications to this market price required at the divisional level that are due to Quantity, or Term Discounts, and expected cash collection difficulties that vary for different classes of clientele and products. The divisional CFO thus creates a factored Market Price that is intended to reflect the cash inflow from contacted account sales for the PS. Next in the planning process the Controllable Costs of the PS were identified that pertain to the following two Direct Cost classes; Materials \& Labor. Even though the MNC used a Hybrid-Activity Based Manufacturing Overhead $[\mathrm{MOH}]$ Allocation model, $\mathrm{MOH}$ was not considered a cost that could or would be modified over the year; whereas the Direct Materials \& Labor components were considered as costs that could be modified through managerial actions. For this reason the next inputs to the cost control model were the Standards for the Materials \& Labor pertaining to the PS. These were not Engineering Standards nor were they what the client called "Hard-Ball" standards - defined as standards that tacitly are based on the assumption that by fortuitous idiosyncratic predisposition every dynamic variation in the production process $\&$ in the economic climate would work to the advantage of the company over time. Rather, their conception of the Standards were practical Standards or realistic expectations. However, the client insisted on using the term Standard Material Component and Standard Labor Component. Additionally, for both the constituent Materials and Labor components of the PS the costs were profiled in Material Bundles and Labor Bundles. This bundling, according to the CFO, who was by training an engineer, allowed various adjustments to both the Materials and Labor bundles as there were different cost elements in each for the bundles. For example, for one of the PS respecting the Standard Material Component $[\mathrm{SMC}]$ there were 73 different component parts that were organized into four bundles used in more or less sequential order over the production process. In this case, the SMC was 4.0. Further, each of these four bundles had a different cost standard depending on the number of component parts in the particular bundle. The Standard Price/Cost [SP] assigned to each of the Bundles was one fourth of the total standard cost. In this case, the SP was $€ 58.50$ as the total standard cost for the 73 component parts represented by the four bundles was given as $€ 234$ [€58.50×4]. We will elaborate on this bundling concept in the illustration of the SCA:DSS. Finally, as for the third cost component the indirect expenses $\mathrm{MOH}$ these are subject to allocation. For this MNC there were two allocation pools pertaining to MOH: The General Pool consisting of: Insurance and general upkeep on the Property, Plant \& Equipment, Heating, Lighting, Janitorial services and the like. Also there was an Activity Based Cost Model [ABC] used to allocate cost to those activities that were unique to the Division and/or Product and that could be tracked to particular Product Sets. Effectively, for the PS there were significant IT-programming costs to fine tune \& maintain the software-links of the PS before as well as after the sale. Given the nature of the PS any allocations of the General $\mathrm{MOH}$ were viewed as interfering with the managerial decision-making prerogatives and so were contentious-points in the evaluation of the relative contribution of the various products and the division. The trade-off arrived between the PS-Division and The Headquarters of the MNC was: NO General-MOH Pool allocations were used in the evaluation of Product-sets at the divisional level. However, HQ did require that the Activity Based Costs be a part of the evolution of divisional or product performance. Therefore in addition to the Direct Materials and Labor tracking, the $\mathrm{ABC}: \mathrm{MOH}$ also figured in the division product performance.

\subsection{Profiled Projections}

The second concept of the SCA: DSS is the formal profiling of the Profit \& Direct Cost components as projections to the end of the year under three profiling scenarios: The Standards Case where all of the planned standards were exactly realized, This was also called the Best Case Profile. The Status-Quo or Stasis Case where the Mid-Term profile is the likely results at YE. Effectively, this Stasis was considered the "Worst-Case" in the sense that there were no actions taken that were effective in putting the production process back on the Standards Trajectory. The third Case was the Effective Action Case that was a What-If platform. Here the division projected the results based upon the $20 \%$ of the time that effective action were in fact able to align the production process with the standard plan.

There was a graphic that was produced by the SCA: DSS that projected the results iterated from $20 \%$ of the 
actions that were effective to $100 \%$ by increments of $10 \%$. We will examine this Graph in the example to be detailed in a later section. This is a major innovation effected by the CFO. The graphical representation of the sensitivity of the cost re-calibration and its effect on the contribution margin to the variation in the components is the realization of the underling rationale of DSS which are useful only if they provide timely and relevant information that will enable effective actions. As the focus of the SCA:DSS is the projected net margin over direct costs the SCA:DSS can profile the tracking of the Profit \& Cost Standard at any time. The CFO decided that profiling the status at the end of the second quarter, termed a Mid-Term Profile, would provide both a reliable production variance analysis [Standard v. Actual] and also provide a reasonable window for effecting corrective actions if needed. Additionally, we emphasized that the after the corrective actions are taken so that their sensitivity-impacts were estimable with some confidence that the effect of the actions be re-estimated using the DSS. To give an example of these three profiling cases consider Table 1 where we have used actual values from the client; we will use these values in the detailed illustration presented subsequently.

Table 1. Client Values used in the Presentation of the Functionalities of the SCA:DSS

\begin{tabular}{cccc}
\hline Projection Section & Best Case [Standards] & Status-Quo Case & Action Result [20\%] \\
\hline Effective Market Price & $\mathbf{6 6 5 0 . 0 0}$ & $\mathbf{6 6 5 0 . 0 0}$ & $\mathbf{\epsilon 6 5 0 . 0 0}$ \\
Product Direct Cost & $€ 234.00$ & 250.49 & $€ 242.18$ \\
Materials & $€ 76.56$ & 105.23 & $€ 90.78$ \\
Labor & $\mathbf{E 3 1 0 . 5 6}$ & 355.72 & $€ 332.96$ \\
Total Direct Factors & $€ 81.80$ & $€ 81.80$ & $€ 81.80$ \\
$\boldsymbol{A B C}: \mathbf{M O H}$ & $€ 392.36$ & $€ 437.52$ & $€ 414.76$ \\
Planned Standard Cost & $€ 257.64$ & $€ 212.48$ & $€ 235.24$ \\
Planned Unit Profit & $\mathbf{3 9 . 6 \%}$ & $\mathbf{3 2 . 7 \%}$ & $\mathbf{3 6 . 2 \%}$ \\
Plan Percent Return & & & \\
\hline
\end{tabular}

In this case, the Standard or Planned case was that there would be a realistic market price for this product - meaning the expected cash flow from overall account sales was $€ 650.00$. The three components of the Direct and Allocated Costs totaled $€ 392.36$ thus giving a contribution of $€ 257.64$ or a percentage of $39.6 \%$ at standard. It is important to recall that this does NOT account for General Overhead, Sales related costs such as product packaging and shipping, or random assurance product testing. At the Mid-term profile the actual costs were $€ 437.52$ per unit and if this were to be the case at the YE then the Actual percent return would be $32.7 \%$ or a short fall of $6.9 \%$ [39.6\% less $32.7 \%$ ]. The last column: Action Results displays the results assuming that corrective actions were $20 \%$ effective for both Materials and Labor. In this case, the "gain" over the Stasis or the Worst case would have been at YE 3.5\% [36.2\% less 32.7\%]. For this last column the SCA: DSS displays the Return Percent for iterations from $20 \%$ to $100 \%$; the values currently in the column are for $20 \%$ success. We shall offer this graphic in the illustration following.

\section{Detailed Illustration of the SCA: DSS Functionalities}

In this section we will give the codex in the logical order of execution. The intention is to give clear details that will enhance understanding of the SCA: DSS. In this regard, we suggest to follow along with the development of the various stages of the SCA: DSS. Additionally, this will aid in modifying the Excel Code. Recall the SCA: DSS is available free as a download without restriction. Finally, almost all of the functionalities are presented in the Excel Formula Worksheet context and so VBA-modules are not needed, the exception being the generation of the Graphic to be presented subsequently.

\subsection{Platform I Computation of the Component Variances: Aggregate \& Disaggregation}

In simple notation: On a Product Unit Basis: Standard Materials [SM]; Actual Materials [AM]; Standard Labor Effort [SL]; Actual Labor Effort [AL]; Standard Price: Materials [SpM]; Actual Price: Materials [ApM]; Standard Wage: Labor Effort [SpL]; Actual Wage: Labor Effort [ApL]

The four Bundle sets for the SM \& AM are $\{$ B1, B2, B2, B4 $\}$. The component parts and the Aggregate Cost assigned to each Bundle was: $\{\mathrm{Bi}[\mathrm{Number}, €]\}:\{\mathrm{B} 1[6, € 40]$; $\mathrm{B} 2[33, € 60]$; $\mathrm{B} 3[20, € 20]$; $\mathrm{B} 4[14, € 114]\}$ In this case, the average price/cost assigned to each bundle was: Total Aggregate Cost, bolded above, over the Bundles divided by the number of Bundles: $€ 234 / 4=€ 58.50$ which is the SpM. As there are four bundles the SM = 4.0. In a similar fashion the Wage rate was the Standard. The Unit for the wage was $€ 17.50$ hours at a work week of 35 Hours which in the country of the MNC was the standard work week by the union contract. Additionally, this wage included university students, mostly at Masters level programs, that were engaged in short term apprenticeship/internship programs. This gives for the SpL: $€ 612.50[35 \times € 17.50$. Labor effort for each unit was fixed at 0.125 of the work week or SL was 0.125. All of this information is entered into the SCA: DSS using VBA Input-Links. Even though there are a number of component elements there are only four inputs required for 
each component. For Example regarding Material \& Labor: $\mathrm{SM}=4, \mathrm{SpM}=€ 58.50, \mathrm{SL}=0.125 \& \mathrm{SpL}=€ 612.50$. As for the actual values these values are drawn from the AIS of the MNC. All of the material and labor activity are Radio Frequency tracked from Inventory regarding Materials and from the Work activity for the Labor Effort. For example, for the actual materials: AM there were more than 73 parts used. In the case presented, there were, on average, 76 parts required so the AM was 4.16438 [4x76/73]. Using these four DM inputs the SCA:DSS produces the following variance analyses:

4.1.1 Platform I Material Profiles \& Analysis of Variance

Materials Variance Aggregate and the Detail Disaggregation Displayed by the SCA:DSS:

$[[\mathrm{SM} \times \mathrm{SpM}]-[\mathrm{AM} \times \mathrm{ApM}]]=[[4 \times 58.50]-[4.16438 \times 60.15]]=[234.00-250.49]=-16.49$

Detail Disaggregation

$[\mathrm{SM}-\mathrm{AM}] \times \mathrm{SpM}=[4.0-4.16438] \times 58.50=-9.62$

$[\mathrm{SpM}-\mathrm{ApM}] \times \mathrm{AM}=[58.50-60.15] \times 4.16438=-6.87$

Re-Aggregation: $[(-9.62)+(-6.87)]=-16.49$

Summary Percentage Profile

Materials Proportional Variance \& Price Proportional Variance

$58 \%[-9.62 /-16.49] \& 42 \%[[-6.87 /-16.49]$

4.1.2 Platform I Labor Profiles \& Analysis of Variance

Labor Variance Aggregate and the Detail Disaggregation Displayed by the SCA:DSS:

$[[\mathrm{SL} \times \mathrm{SpL}]-[\mathrm{AL} \times \mathrm{ApL}]]=[[0.125 \times 612.50]-[0.162 \times 649.57]]=[76.56-105.23]=-28.67$

Detail Disaggregation

$[\mathrm{Sl}-\mathrm{Al}] \times \mathrm{SpL}=[0.125-0.162] \times 612.50=-22.66$

$[\mathrm{SpL}-\mathrm{ApL}] \times \mathrm{AL}=[612.50-649.57] \times 0.162=-6.01$

Re-Aggregation: $[(-22.66)+(-6.01)]=-28.67$

Summary Percentage Profile

Labor Proportional Variance \& Price Proportional Variance

$79 \%[-22.66 /-28.67] \& 21 \%[[-6.01 /-28.67]$

This Standard v. Actual Analysis of Variance is Initial Platform presented by the SCA:DSS.

\subsection{Platform II the Production Activity}

This profile is the aggregate values of the production activities-i.e., the Equivalent Units of Total Production [EUP]. For this platform the DM is asked for the: Beginning Inventory [BI] \& The Percentage of Completion [PcBI], the Number of goods transferred to Finished Goods during the year [T] and the Ending Inventory [EI] \& The Percentage of Completion [PcEI]. This platform used the actual values that were measured/uploaded at the Mid-Term [31May].

Platform II The EUP are computed as:

$$
\begin{aligned}
& \mathrm{EUP}=\mathrm{BI} \times(1-\mathrm{PcBI})+(\mathrm{T}-(\mathrm{BI} \times(1-\mathrm{PcBI})))+\mathrm{EI} \times \mathrm{PcEI}) \\
& 1,264 \times(1-22 \%)+(34,293-(1,264 \times(1-22 \%)))+923 \times 47 \%) \\
& 985.92+33,307.08+433.81=\operatorname{ROUNDUP}(34,726.81,0)=34,727
\end{aligned}
$$

\subsection{Platform III the Profiler}

This is where the computations of interest to the DM regarding effecting corrective actions are produced and thus was her focus of the SCA: DSS. In this case, the DM enters the total units planned for the year [TuP]: TuP was 56,010. Recall that we are at the Mid-Term and the SCA:DSS will generate the info-set that will be used to profile the three cases as presented in Table 1. The first set of computation in this regard produces the following:

4.3.1 Details Useful in Forming the Decision Regarding Corrective Actions: Materials [Monetary Value]

Required Bundles at Standard: $\mathrm{TuP} \times \mathrm{SM}=56,010 \times 4=224,040$.

Total Projected Cost at Standard: $224,040 \times 58.50=€ 13,106,340$. 
Actual Cost to 31May: $4.16438 \times 60.15 \times 34,727=€ 8,698,677.92$.

Production Cost Remaining to achieve the plan: $€ 4,407,662.08$ [€13,106,340-€8,698,677.92]

Recalibration of the cost per bundle to achieve the initial plan given the mid-term results:

$€ 51.775=[4,407,662.08 /[(56,010-34,727) \times 4.0]]$

In this case the SCA:DSS summarizes the Materials profile as:

The Reduction in the cost from 58.50 to 51.775 or a reduction of 6.725 or a reduction of $11.5 \%$ will be needed to arrive at the planned profit relationships at $Y E$. [Rm=6.725]

This part of the SCA:DSS answers the question: Given that we are at 31May in the production process and we have identified that we are not on track to achieve the plan, how much would the costs of the Bundles have to be reduced to achieve the plan assuming that we maintain the SM at 4.0.?

Point of clarification. The CFO did not want to vary both the material \& the cost components. She decided to fix the materials component at standards and recomputed the price component as this seemed the simplest and most effective was to put production process "back on track". To us this also seemed the best way to reestablish control; thus this is the option built into the SCA:DSS. For example, given that the current costs are $€ 8,698,630.33$ if we add to this the production require to achieve the plan which is: 21,283 [56,010-34,727] projected at the standard of 4.0 bundles, then the price is recalibrated at 51.775 will put the system back on target to arrive at the projected cost at standard as noted above of: $224,040 \times 58.50=€ 13,106,340$. Specifically,

$€ 13,106,340.00=[€ 8,698,677.92+[21,283 \times 4.0 \times € 51.775]]$

4.3.2 Details Useful in Forming the Decision regarding Corrective Actions: Labor [Monetary Value]

For the Labor Component in a similar fashion we have the following:

Required Bundles at Standard: TuP $\times \mathrm{SL}=56,010 \times 0.125=7,001$

Total Projected Cost at Standard: $7,001 \times 612.50=€ 4,288,265.63$

Actual Cost to 31May: $649.57 \times 0.162 \times 34,727=€ 3,654,334.02$

Production Cost Remaining to Achieve the Plan: $€ 633,931.61$ [€4,288,265.63-€3,654,334.02]

Recalibration of the Cost per Bundle to achieve the Initial Plan Given the Mid-Term Results:

$238.28656=[633,931.61 /[(56,010-34,727) \times 0.125]]$

In this case the SCA:DSS summarizes the Labor profile as:

The Reduction in the cost from 612.50 to 238.29 or a reduction of 374.21 or a reduction of $61.1 \%$ will be needed to arrive at the planned profit relationships at $Y E .[\mathrm{R} l=374.21]$

Now that the two major recalibration are known, then the three DM-profiles can be examined.

\subsection{Platform IV Profile Information Set and What-If Testing}

Referencing Table 1 let us detail the computations therein. The Best Case column is the Plan; this of course is the benchmark for the DM. The projected costs for Materials of $€ 234.00$ and Labor of $€ 76.56$ give the Direct Controllable costs as detailed above. The ABC: $\mathrm{MOH}$ of $€ 81.80$ is added in to form the planned standard cost of $€ 392.36$ which produces a planned profit per unit of 257.64 or a $39.6 \%$ return on the expected cash flow from sales. A clarification regarding the $\mathrm{ABC}$ : $\mathrm{MOH}$ component. The $\mathrm{CFO}$ used the decision making information for the three cases without modifying the value of the $\mathrm{ABC}$ : $\mathrm{MOH}$ component. We discussed with her why she regarded this as invariable. Her logic was that most of the $\mathrm{ABC}$ allocations were IT related and determined by HQ at the beginning of the planning process. The HQ: IT-division is the company-wide support most of whom worked "on-line" at the HQ in a different city. She indicated that in the last two years she has never seen any of the HQ-IT staff nor had any occasion to interact with them in any forum. At the beginning of the planning period $\mathrm{HQ}$ indicates the total ABC: MOH charge which is usually on the order of four million dollars as her division is highly automated and uses many robotic integrated modules. There is never a change in this amount. In our experience, this is the norm in the IT context.

As for the Stasis Case, the third column, the computation of the Materials and the Labor is simply the actual results at the Mid-Term assumed to be the case also at YE. This is sort of the Stasis-Case scenario relative to making the modifications. Therefore the range for the decision making profile benchmarking for the last column called the Action Profile is: 7.3\% [32.3\% to 39.6\%]. As this Action Profile is the critical feature of the SCA:DSS, we programmed this to produce a trajectory graphic with iterations starting at $20 \%$ corrective effectiveness 
ranging to $100 \%$ effectiveness as presented in Figure 1. Recall that effectiveness refers to the corrective actions to move the actual result at the Mid-Term to arrive at the Plan at YE. The important implication of this is that the recalibrated amount can be used directly to project the yearly results NOT just the incremental change from the Mid-Term results. The programming of this What-If modification is as follows:

\subsubsection{For Materials}

In the case of the following scripting IP is the iterated percentage [IP] of the success of the actions taken to reduce the cost. This iterated range of IP is 20\% to 100\%. In the example following IP is set to $20 \%$.

Profiled Iterated $\operatorname{Cost}(\mathrm{IP})=[[\mathrm{AM} \times \mathrm{ApM} \times \mathrm{EUP}]+[\mathrm{SM} \times(\mathrm{TuP}-\mathrm{EUP}) \times[\mathrm{SpM}-(\mathrm{R} m \times \mathrm{IP})]] / \mathrm{TuP}$.

Profiled Iterated $\operatorname{Cost}(20 \%)=€ 242.18$ and is presented for illustration in monetary values as follows:

$[[4.16438 \times 60.15 \times 34,727]+[4.0 \times(56,010-34,727) \times[58.50-(6.725 \times 20 \%)]] / 56,010$.

$[8,698,677.92+[4.0 \times 21,283 \times[58.50-1.345]] / 56,010$

$[8,698,677.92+[4.0 \times 21,283 \times[57.155]] / 56,010$

$[[8,698,677.92+[4,865,719.53]] / 56,010$

$€ 242.18$ as is reported in Table 1.

In this case of a $20 \%$ reduction in the cost of the material Bundle, if the corrective efforts to move from a Bundle cost of 58.50 to 57.155 where 57.155 is computed as:

The total reduction needed as computed by the SCA:DSS is $€ 51.775$. This recalibration point of $€ 51.775$ can be thought of as: $[58.50-6.725 \times$ IP]. If IP $=20 \%$ then we have $[58.50-6.725 \times 20 \%]$ or [58.50 - 1.345] which is $€ 57.155$.

Therefore, the Material cost per unit would be $€ 242.18$ assuming that the cost reduction initiatives were $20 \%$ successful. Compare this to the best cases of $€ 234.00$ or the Stasis case [No Action taken] of $€ 250.49$. The same is assumed true for the corrective efforts respecting Labor where a $20 \%$ success rate creates an overall Labor cost of $€ 90.78$ compared to the Best Case of $€ 76.56$ to the Stasis Case of $€ 105.23$.

\subsubsection{What-If Variable Cases}

As noted above, in the adaptive context DM must be cognizant of the temporal trade-off in effecting actions. For this reason, we programmed a What-If platform where DM may enter a corrective action for Materials and a different corrective action for Labor as there may be different adaptive time frames for each. The CFO considered this functionality to be the key decision making feature of the SCA:DSS. [Note this is not the same as the iterated graphic as presented in Figure 1 where both components are iterated at the same values.] For example, assume that the DM has planned to take corrective actions which will result in $35 \%$ success rate respecting Materials and for Labor 53\% these values are entered using the VBA InputBox function. The results is that the percent of profit anticipated after the corrective actions are effected is 37.3\% compared to the Planned of $39.6 \%$, Stasis of $32.7 \%$ and $20 \%$ corrective actions of $36.2 \%$. Additionally the SCA:DSS computes and presents the total profit expected given the corrective actions as: $€ 13,590,204.83$. Finally the Shortfall respecting the Standard Plan is competed as: $€ 840,071.54$ and the Gain respecting Stasis of: $€ 1,689,076.64$

For complexness these computations presented in detail. In the SCA:DSS they are only presented in final values.

Details of the Specific Case What-If Platform [There are slight computational differences between the computations following that are presented in monetary units and those produced by the SCA:DSS as Excel forms computations to 25-Decimal places. We used the monetary values taken from the SCA:DSS]:

Materials Corrective rate of $35 \%$

$[[4.16438 \times 60.15 \times 34,727]+[4.0 \times(56,010-34,727) \times[58.50-(6.725 \times 35 \%)]] / 56,010$.

Per Unit Material cost for the Standard of 4 Bundles: $€ 240.64$

Labor Corrective Rate 53\%

$[[0.162 \times 649.57 \times 34,727]+[0.125 \times(56,010-34,727) \times[612.50-(374.21 \times 53 \%)]] / 56,010$.

Per Unit Labor cost for the Standard of 0.125 Effort: $€ 84.92$

Percent Profit after corrective action are realized

$[650.00-[240.64+84.92+81.80]] / 650.00$

$242.64 / 650.00=37.3 \%$ 
Monetary Gain relative to Stasis

[242.64-212.48] ×56,010=€1,689,076.64

Shortfall Relative to the Standard Plan

$[257.64-242.64] \times 56,010=€ 840,071.54$

Both the specific change platform and the iterated figures may be used to probe the change possibilities considering the various interacting agents in the system's logistic. One may start with the iterated graphic to examine the sensitivity of the configured production space. This will be a major decision aid in deciding the cost of corrective efforts relative to the likely benefits. For example, if the slope of the Graphic of the What-If analysis in Figure 1 is relatively steep for any of the particular incremental changes then this would encourage corrective actions using the Specific What-If platform of the DSS. For example, using the graph as an impetus for taking corrective action, the DM can use the Specific What-If platform to create a graphic where the corrective actions are iterated starting at $20 \%$ effective for both to $100 \%$ effective. This may be useful in conjunction with the specific changes illustrated above.

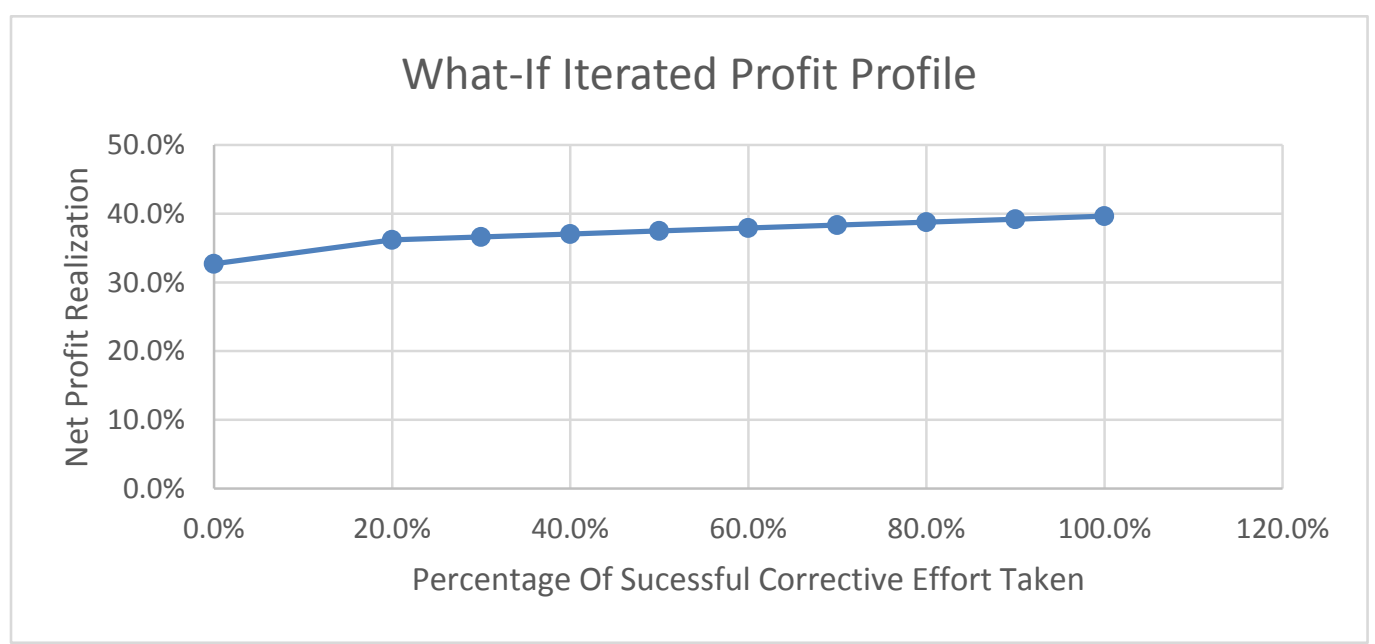

Figure 1 . The iterated What-If graph $20 \%$ to $100 \%$ in the case produced by the SCA: DSS

\section{Summary, Suggestions and Outlook}

\subsection{Summary}

This consulting engagement produced a SCA:DSS that was developed to meet the needs of the decision-making group; it is true that these needs are idiosyncratic. There were a number of these particular design features and events that we wish to note:

The Actual v. Standard analysis of variance that is the first platform and is the usual fare presented in the Cost Accounting texts [See Datar \& Rajan, 2017, Ch7] was rarely, if ever used, by the CFO or those involved in the evaluation for monitoring and control at the divisional level. The sentiment seems to be that the important information was only the differences: [Actual v. Standard] for the Component and the Cost dimensions. The arrangement of these differences by multiplying them by factors so that they reconcile to the overall difference seemed to be an artifact of the analysis and did not add any real information of a decision-making nature. In the adaptive context the component analysis of variance is a standard feature of most costing systems and the one detailed by Horngren. However, in this study context such a standard unraveling of the overall variances was not deemed useful in effecting adaptive dynamic holistic change for this MNC.

The divisional decision-makers thought that any allocated costs, including those that were $\mathrm{ABC}$ allocations, were nuisance factors of headquarters and were of no practical concern to the divisional decision makers. This violates most of the 14 Points offered by Deming and was a major impediment to effective corrective actions.

Allocations of $\mathrm{MOH}$ were characterized by the CFO as "An Indication of how much effort one puts into the negotiation of these arbitrary allocations with the DMs at HQ." Further, she suggested that "the more profitable divisions subsidized the allocations for "favorite" divisions or "start-ups" or division with "new products".

The What-If analysis, both the Graphical and the Specific components of the SCA:DSS, were considered the key features and are consistent with the issues raised by Deming and his elaborators as noted above relative to effecting actions in a reasonable proximal timeframe. 
Corrective actions were almost exclusively formed around the value of the recalibrated planned profit per unit differential between the Stasis case and the $20 \%$ effect case.

Rarely was the target more than a $20 \%$ effort. The CFO mentioned many times that the time frame for a $20 \%$ effort was reasonable. She thought that $100 \%$ success was not attainable in a timeframe that was practical.

There was never structured follow-up at year-end at the divisional level or at HQ as to the effectiveness of the corrective actions actually taken and their effect - that is to say a Post-Audit of the actions taken The success measure therefore was never the actual monetary effect but rather seemed to be simply the intention to try to effect a change and the various actions taken and not their actual effect on the monetary values. This seems to be a failure of the Headquarters to create the tone-at-the-top that encourages or perhaps requires a learning loop in the system. This failure is a marked impediment to creating a holistic context.

There was never any discussion of modification of the Factored Market price in conjunction with the Cost Modifications as a way to achieve the Plan. This was interesting and curious as the Market Price was set for the first two quarters and sometimes was modified due to economic climate $\&$ outlook charges.

We re-connected with this MNC three years after this engagement. The CFO had moved on to another MNC. The SCA:DSS was no longer used in the division by the current CFO.

\subsection{Suggestions Given this Debrief We Offer}

Addressing the Deming Issues This debrief details the plethora of failures to realize a holistic context for this MNC. One possible issue was that there were many bonus arrangements over the MNC. Various worker groups had negotiated union contracts that had different bonus protocols that in total seemed to create dysfunctions in motivating a common vision of cooperation and the systemic trade-offs needed to realize an overall goal. Additionally, the salaried individuals [basically the management of the various Divisions and HQs] had different contract-bonus arrangements that were not public information so we could not read these contracts. Perhaps there needs to be "unified" monetary rewards over all the wage and salary groups that encourage all the agents in the MNC to act in the overall monetary best interest so that everyone is better off relative to what they would have been if they had sub-optimized their individual monetary reward functions. This is certainly a daunting requirement. However, unless specific software or shareware, such as the SCA:DSS, is a formal and required part of the divisional evaluation process and also impacts at least the decision makers at the HQ division there is no reason to expect that such software will find its way into the decision-making system of the firm.

It is to be expected that the DSS need to be developed to meet the needs of the users. In this regard, we held a number of developmental sessions that almost exclusive benefited from creative developmental suggestions.

Over time there will be new members of the divisional team. Their developmental suggestions should also be solicited in regularly scheduled re-deployment and feedback workshops.

One issues that was raised by this consulting engagement that was not addressed was the time frame for corrective action. While it was true that the CFO's selection of the end of the second quarter of the year seems like a reasonable choice, the actionable information, even though available as soon as the SCA:DSS was parameterized, will require time to effect changes in the costs. The time to actually effect cost modifications is very likely to extend well into the next quarter. In this regard, it would be an excellent idea to re-visit the re-calibration when the actual cost modifications were to be known. This fits well with the experience of Toyota as documented in the excellent case study by Parnaby \& Towill (2009) where they note:

Those who follow gurudom and subsequently believe that instantaneous across-the-board success is achieved by TPS orientated change management programmes are due for a rude awakening (Schonberger, 2007). For example, it is claimed that realistically Toyota took some 20 years to fully develop and implement just-in-time production. Even when armed with specialist TPS fore knowledge it is still a lengthy procedure, which Ohno (1988) suggested may well be a ten year hard slog to play catch-up. Consequently, any hoped for "quick hits" can usually only be achieved against the right organisational-, motivational-, and knowledge-based background.

This reinforces the importance of the holistic commitment - to wit: unless the whole enterprise is supportive of the action logistic and the required time frame for effective execution there will be Deming issues and failures created in realizing the corrective actions needed to re-take control. The developments of holistic integrated monitoring for corrective actions of the mid-1990s are well detailed by Gadd (1995).

For us, this was an academic consultation. This means effectively that there was no cost outlay by the MNC or Division for the development of the DSS. Interestingly, this seemed to create a "lack of real commitment" at the 
firm level. Perhaps there was the belief that as there was no cost there was no value!!! That is to say that we were doing this "only" for our academic benefit. Perhaps, all consultations should require some monetary cost on the part of the organization to engender commitment.

The SCA: DSS that we developed mirrored the needs of the individuals in this particular division. Therefore, effectively the SCA: DSS is useful as an illustration of the possibility of corrective action in their production stream and the debrief information-set acts as issues that may constrain the effective utilization such an adaptive modeling system. We suggest that Cost Accounting courses could benefit from demonstrating Corrective Action Profiling and What-If Re-calibrations as they may impact the holistic organizational context. These paradigms, we offer, should be the design feature for publishing organizations as Pearson[https://www.pearson.com/us/], Wiley[https://www.wiley.com/en-us] \& McGraw-Hill[https://www.mheducation.com/] to mention a few. If these major publishing organizations move to encourage authors to treat these paradigms authors may follow suite. The SCA: DSS would be ideal for this purpose and could integrate very seamlessly with themes that offer these adaptive and holistic features as text illustrations. For example, we used a version of the SCA:DSS in the Cost section of the course that we taught. The students, most of whom were addicted to video games, seemed to be drawn to use the SCA: DSS and some actually re-programmed some sections. In summary, while most of the texts speak to the need to holistic imperatives, rarely are details of the practical aspects used to illustrate how to effect such system's synergy in adaptive costing. It is encouraging, indeed, that academic presentations of these paradigms are now beginning to find currency in the literature as the Venzor \& Ivanov (2017) work illustrates. To be clear: there needs to more integration between Theory and the Practical Context where one should expect to see Theory IN Application and this needs to be presented in the text presentations.

As for adopting the SCA: DSS in an actual production facility: One could start with the SCA: DSS platform and in focus groups and developmental workshops re-tool the SCA:DSS to fit the particularities of the firm. We suggested this to the CFO who replaced the CFO that was our point-person for the academic consultation. There was no follow-up on these ideas.

\subsection{Outlook}

We believe that there are two critical aspects of any SCA:DSS: (i) There MUST be feedback well in advance of the end of the year and, (ii) adaptive corrective actions should be required and evaluated in the holistic context discussed by Deming and offered as a pre-condition to effective corrective actions. The SCA: DSS is the first stage in the process of change as it marks variance form the plan. However, as we have seen in the debrief and suggestions there is a need for commitment at all levels before the SCA: DSS can achieve it potential.

We offer the SCA: DSS as a flexible DSS that is easily modified to meet the needs of users as a free download without restrictions on its use. Also, we are happy to discuss any and all implementation issues either in the development as an integrated module for a Cost Accounting course or as a developmental platform for a production facility, or as a useful tool in the panoply of the CPA as a consultant. In this regard, Babicky (1996) notes:

The CPA can play an important role in the benchmarking for the organization. Benchmarking is the process of determining critical success factors and making comparisons to the best in the class. Finding the appropriate benchmark candidates and developing the in-house data for comparative purposes is an important undertaking.

Finally, there needs to be more attention paid to adaptive holistic controls in costing systems. We hope that publishers and authors move with dispatch in this direction by providing practical illustration to motivate these critical paradigms as part of the delivery to the next generation of the managers-our students.

\section{Acknowledgments}

Thanks and appreciation are due to: Dr. H. Wright, Boston University: Department of Mathematics and Statistics, the participants at the SBE Research Workshop at SUNY: Plattsburgh [In particular: Professor Dhimitri Qirjo of the Economics' Group], Mr. Manuel Bern, Chief of Internal Audit: TUI International, GmbH, Hannover, Germany, and the reviewers of the International Business Research journal for their careful reading, detailed comments, and suggestions.

\section{References}

Ackoff, R. (1967). Management misinformation systems. Management Science, 14, B147-B156. https://doi.org/10.1287/mnsc.14.4.B147

Babicky, J. (1996). TQM and the role of CPAs in industry. The CPA Journal, 66, 69. 
Brimson, J. (2011). Management paradigm: The untapped power of process laws. The Journal of Corporate Accounting \& Finance, 22, 33-44. https://doi.org/10.1002/jcaf.20673

Datar, S., \& Rajan, M. (2018). Horngren's cost accounting: A managerial emphasis. Pearson, New York, NY. USA. ISBN 13: 978-0-13-447558-5

Deming, W. E. (1992). Out of the crisis. Journal of Leadership and Management, 1, 57-66. Originally: Deming, W. Edwards. (1986). Out of the Crisis. Cambridge, MIT Press Cambridge, USA: ISBN: 0911379010

Deming, W. E. (1993). The new economics: For industry, government, education. MIT, Cambridge, MA. USA 1993. ISBN-13: 978-0-262-54116-9

Deming, W. E. (2005). A System of Profound Knowledge, in: John C. Wood and Michael C. Wood (eds), W. Edwards Deming: Critical Evaluations in Business and Management, Volume 1. Taylor and Francis. USA. ISBN 10- 0415323878; ISBN 13- 9780415323871

Denzau, A., Minassians, H., \& Roy, R. (2016). Learning to cooperate: Applying Deming's new economics and Denzau and North's new institutional economics to improve interorganizational systems thinking KYKLOS , 69, 471-491. https://doi.org/10.1111/kykl.12117

Gadd, K. (1995). Business self-assessment: A strategic tool for building process robustness and achieving integrated management. Business Process Re-Engineering \& Management Journal, 1, 66-85. https://doi.org/10.1108/EUM0000000003894

Linnenluecke, M., Birt, J., \& Griffiths, A. (2015). The role of accounting in supporting adaptation to climate change. Accounting and Finance, 55, 607-625. https://doi.org/10.1111/acfi.12120

Ohno, T. (1988), Toyota production system: Beyond large scale production, Productivity Press, Cambridge, MA. USA ISBN-13: 978-0-915299-14-0

Parnaby, J., \& Towill, D. (2009). Exploiting the concept of a manufacturing system part II: Principles and origins of the managing-by-projects engineering change methodology. Journal of Manufacturing Technology Management, 20, 1047-1069. https://doi.org/10.1108/17410380910997209

Schonberger, R. J. (2007). Japanese production management: an evolution - with mixed success. JOM, 25, 403-419. https://doi.org/10.1016/j.jom.2006.04.003

Venzor, A., \& Ivanov, S. (2017). Strategies to improving corporation X: Practical application of Abilene paradox theory of Jerry B. Harvey and leadership theory of W. Edwards Deming. The International Journal of Organizational Innovation [SecA], 9, 105-110.

Zugno, M., \& Conejo, A. (2015). A robust optimization approach to energy and reserve dispatch in electricity markets. European Journal of Operational Research; 247, 659-671.

https://doi.org/10.1016/j.ejor.2015.05.081

\footnotetext{
${ }^{\mathrm{i}}$ As there were more than minor legacy issues with the initial version of the SCA:DSS we have re-programed the components of the SCA:DSS into the Microsoft Office Suite: Excel ${ }^{\mathrm{TM}} .2013$. This version should migrate to future versions of Excel as well as to Open-Office ${ }^{\mathrm{TM}}$.
}

\section{Copyrights}

Copyright for this article is retained by the author(s), with first publication rights granted to the journal.

This is an open-access article distributed under the terms and conditions of the Creative Commons Attribution license (http://creativecommons.org/licenses/by/4.0/). 